\title{
THE EXPECTED IMPACT OF MULTIPLE SCATTERING ON ATLID SIGNALS
}

\author{
D.P. Donovan
}

Royal Netherlands Meteorological Institute (KNMI) P.O. Box 201, 3730 AE, De Bilt, The Netherlands. Email:donovan@knmi.nl

\begin{abstract}
ATLID stands for "ATmospheric LIDar" and is the lidar to be flown on the Earth Clouds and Radiation Explorer (EarthCARE) platform in 2018. ATLID is a High-Spectral Resolution (HSRL) system operating at $355 \mathrm{~nm}$ with a narrower field-of-view and lower orbit than the CALIPSO lidar. In spite of the smaller footprint multiple-scattering (MS) will have an important impact on ATLID cloud signals and, in some aspects, the accurate treatment of MS will be more important for ATLID than CALIPSO. On the other hand, the relationship between integrated backscatter and integrated MS induced depolarization in water clouds will be similar between ATLID and CALIPSO indicating that a CALIPSO-like strategy for cloud-phase identification can be successfully applied to ATLID.
\end{abstract}

\section{INTRODUCTION}

It has long been known that in order to accurately model the lidar signal from clouds that the contribution of multiply scattered photons must be taken into account. This generally holds true for ground-based and aircraft-based lidar systems and is most certainly true for space-based systems. The multiply scattered return depends on both the instrument characteristics and the atmospheric optical properties. In particular, the degree of multiple-scattering (MS) depends on the cloud extinction profile, the distance from the lidar, the wavelength of the lidar, the laser divergence, the receiver field-of-view (fov) and the angular width of the forward scattering lobe associated with the particle scattering which in turn is related to the cloud/aerosol cross-sectional area. The last point is of particular importance and can lead to significant MS effects even at low optical depths. This is the case if the particle sizes are sufficiently large leading to a forward scattering lobe (which in the limit contains $1 / 2$ the scattered energy) which is angularly narrow in comparison to the lidar fov.
ATLID is a HSRL lidar to be flown on the ESA/JAXA Explorer (EarthCARE) platform planned for launch in 2018 [1]. ATLID operates at a wavelength of $355 \mathrm{~nm}$ with a telescope fov of 0.065 mrads (full-angle). EarthCARE's orbit will be about $400 \mathrm{~km}$. CALIPSO orbits at a higher altitude (about $700 \mathrm{~km}$ ) and has a large fov (0.13mads) leading to larger footprint. In spite of ATLID's smaller footprint (about 30 vs 90 meters) MS effects will still be as (or even more) important than for CALIPSO.

In this paper, we will discuss the impact of multiple-scattering on ATLID signals in ice and water clouds and how it can be accounted for and even exploited.

\section{Multiple Scattering in Ice Clouds}

The simplest manner in which MS effects can be modelled is by adjusting the effective particle extinction, i.e.

$$
\beta_{a t}(z)=\beta \exp \left[-2 \eta \int_{0}^{z} \alpha\left(z^{\prime}\right) d z^{\prime}\right]
$$

where $z$ is the range from the lidar, $\beta_{a t}$ is the attenuated backscatter, $\beta$ is the atmospheric backscatter coefficient, $\alpha$ is the extinction coefficient and $\eta$ is the MS coefficient. This approach, originally due to Platt [2], is simple but suffers from a number of limitations. For ATLID the inability to accurately model the backscatter below semi-transparent cloud layers is a serious limitation.

Example results of $\mathrm{MC}$ calculations applied to idealized cirrus cloud are shown in Fig. 1. Here clouds of two different Optical Thickness (OT) comprised of hexagonal ice crystals [3] with modified-gamma particle size distributions and associated mean diameters (with diameter being identified with the crystal maximum length) of 10 and 50 microns have been used. The corresponding effective radius ( $\mathrm{R}_{\text {eff= }}$ $0.75<\mathrm{Vol}>/<\mathrm{A}_{\mathrm{c}}>$ ) values are shown in the 


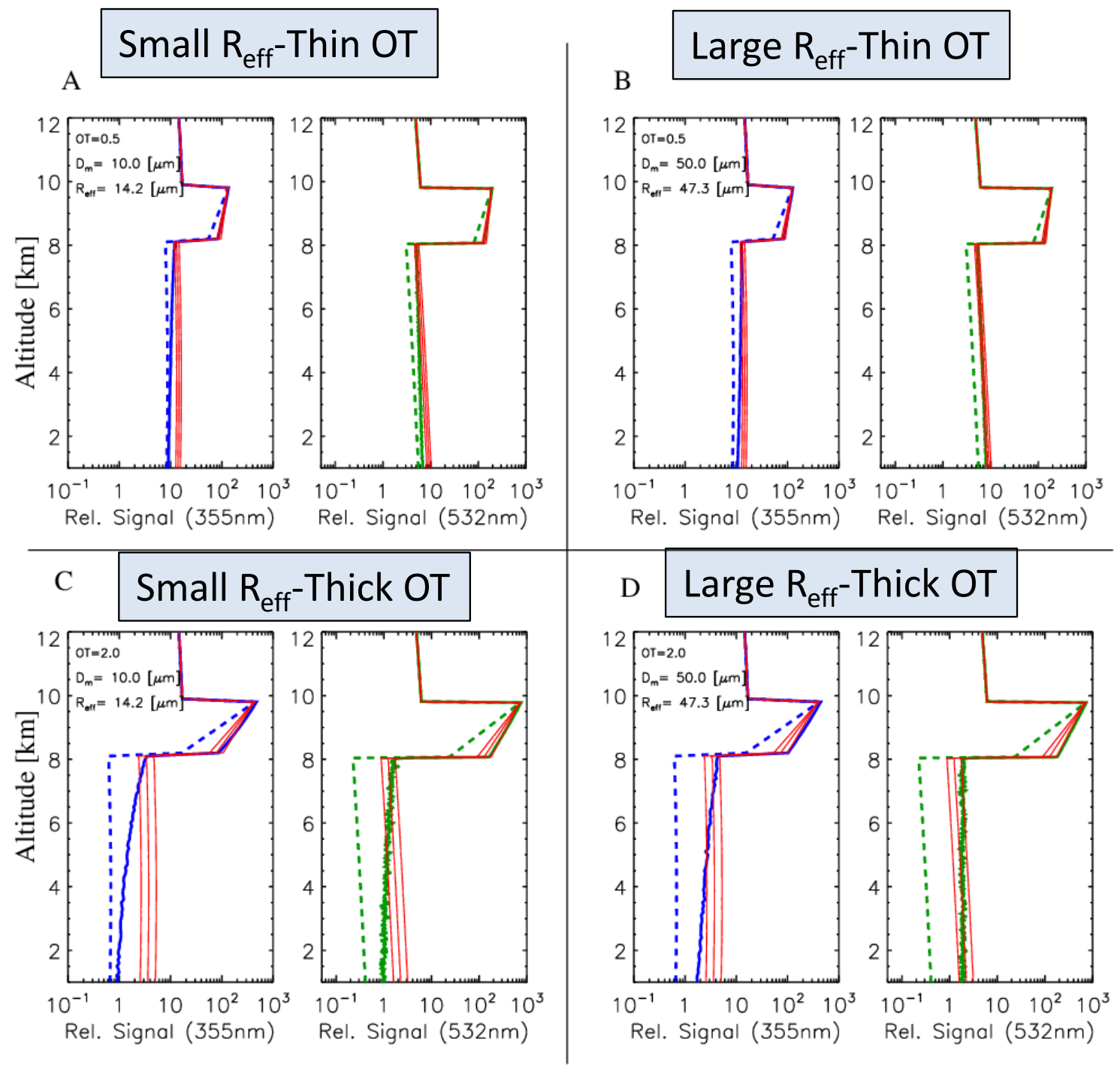

Figure 1: Example results of MC lidar radiative transfer simulations for ATLID (blue-lines), CALIPSO (Green-lines) compared to the expected Single-Scatter signal levels (Dotted Lines) and Eq. 1 with $\eta=0.4,0.5$ and 0.6 (Red-Lines). Panel $A, O T=2$ and $D_{m}=10.0$ microns Panel B: Cirrus $O T=2$, $D_{m}=50.0$ microns and $R_{\text {eff }}=47.3$ microns, Panel $C$ : Cirrus OT=0.5, $D_{m}=10.0$ microns, Panel D: Cirrus $O T=0.5, D_{m}=50.0$ microns and $R_{\text {eff }}=47.3$ microns.

panels. Monte-Carlo (MC) lidar radiative transfer results are shown as well as results corresponding to single-scattering only and the result of applying Eq. 1 with 3 different values of the multiplescattering coefficient $(0.4,0.5,0.6)$. The $\mathrm{MC}$ calculations were performed using the lidar component [4] of the EarthCARE simulator (ECSIM) [5]. ATLID has three channels, a copolar 'Mie' channel, a co-polar Rayleigh channel and a cross-polar Rayleigh+Mie channel. The ATLID results shown in Fig. 1 correspond to the sum of the three channels.

Referring to Fig. 1, it can be seen for this example that Eq. 1 can be used to accurately model the lidar return within the cloud for both CALIPSO and ATLID. However, in the case of ATLID, the Rayleigh signal below cloud base displays a clear "decay" towards the single-scatter signal level. The decay is not as noticeable in the CALIPSO simulations; the decay only becomes noticeable for the larger OT cases especially in the small particle case.

The behavior of the tails may be understood by considering the effective divergence of the multiple-scattered light emerging from the cirrus cloud. With reference to Fig.2, in the case of CALIPSO the instrument footprint is such that only a small about of the multiply scattered light 'leaks' outside of the receiver cone leading to a long decay distance. For ATLID, the smaller fov 

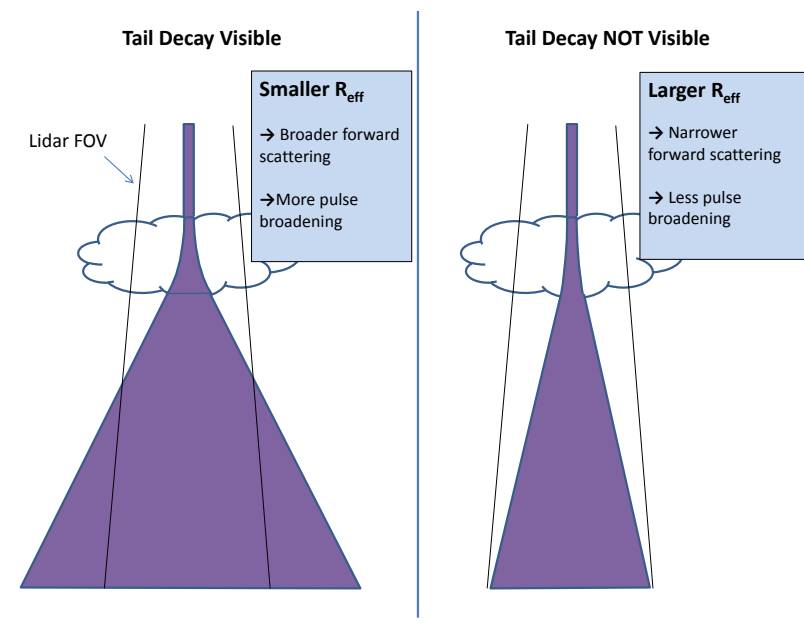

Figure 2: Schematic depiction of the beam broadening caused by MS and how it can lead to visible tails under cirrus clouds.

means that much more of the multiple-scattered light emerging from the cirrus cloud base leaves the receiver cone leading to shorter decay time.

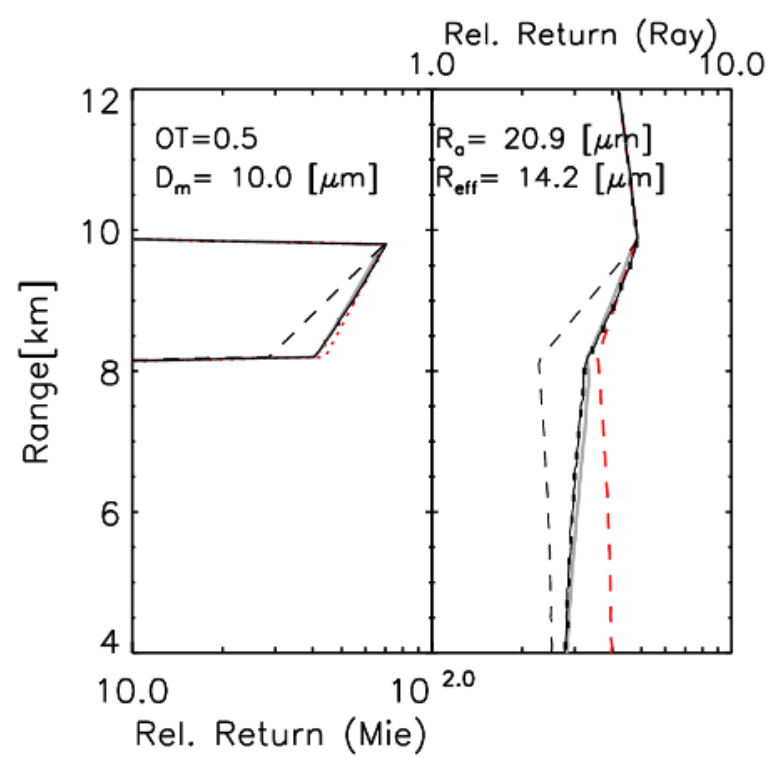

Figure 3: (Left) Mie co-polar and (Right) Rayleigh channel co-polar returns for an ice cloud of an optical thickness of 0.5 and an effective radius of 14.2 microns. Black solid: ECSIM MC results. Dashed Black: Single scattering results. Solid Grey: Hogan's model results and Red-Dashed Eq.1 with a MS factor of 0.45 .

Thus, somewhat counterintuitively, for the case of targets below cirrus clouds, multiple-scattering effects will be more noticeable for ATLID than CALIPSO. In order for ATLID to retrieve accurate extinction and backscatter estimates below semi-transparent cirrus the MS tails must be accounted for.

Since Eq.1 cannot model below cloud tails, more sophisticated approaches are needed for ATLID. Fortunately, fast and accurate analytical approaches exist. One of these approaches has been developed by Hogan [6]. An example comparison between the results of an ECSIM MC calculation and the corresponding result from the fast semi-analytical model of Hogan [6] together with the result of applying Eq.1 is shown in Fig. 3. An idealized cirrus cloud with an optical thickness of 0.5 was used with a particle size mode diameter $\left(\mathrm{D}_{\mathrm{m}}\right)$ of 10 microns giving rise to the indicated values of effective radius $\left(\mathrm{R}_{\text {eff }}\right)$ and mean area radius $\left(R_{a}\right)$. Here it can be seen that all three approaches perform well within the confines of the cirrus cloud. However, the Rayleigh return below the ice cloud is not well-modelled by the Platt approach. The approach of Hogan, however, provides a reasonably accurate match to the MC calculations even below the cloud.

\section{Depolarization in Water Clouds}

The scattering properties of liquid water cloud droplets can be modelled by assuming that the droplets are perfect spheres and applying Mie theory. Under this assumption and assuming single-scattering only, no cross-polar returns from the cloud will exist. However, the occurrence of MS will give rise to a significant cross-polar return from water clouds. Fig. 4 shows the results of $\mathrm{MC}$ calculations for various idealized water clouds for both CALIPSO and ATLID conditions. This figure illustrates that the observed relationship between integrated attenuated backscatter and depolarization for CALIPOS can be replicated by the ECSIM MC model and that the general form of the relationship expected for ATLID will be similar to that of CALIPSO. This indicates that, similar to CALIPSO, this relationship can be used by EarthCARE as a robust means of distinguishing between ice and water clouds.

\section{Conclusions}

The results of extensive MC ice-cloud calculations suggest that the Platt approach will not be sufficient, in general, to model the below- 

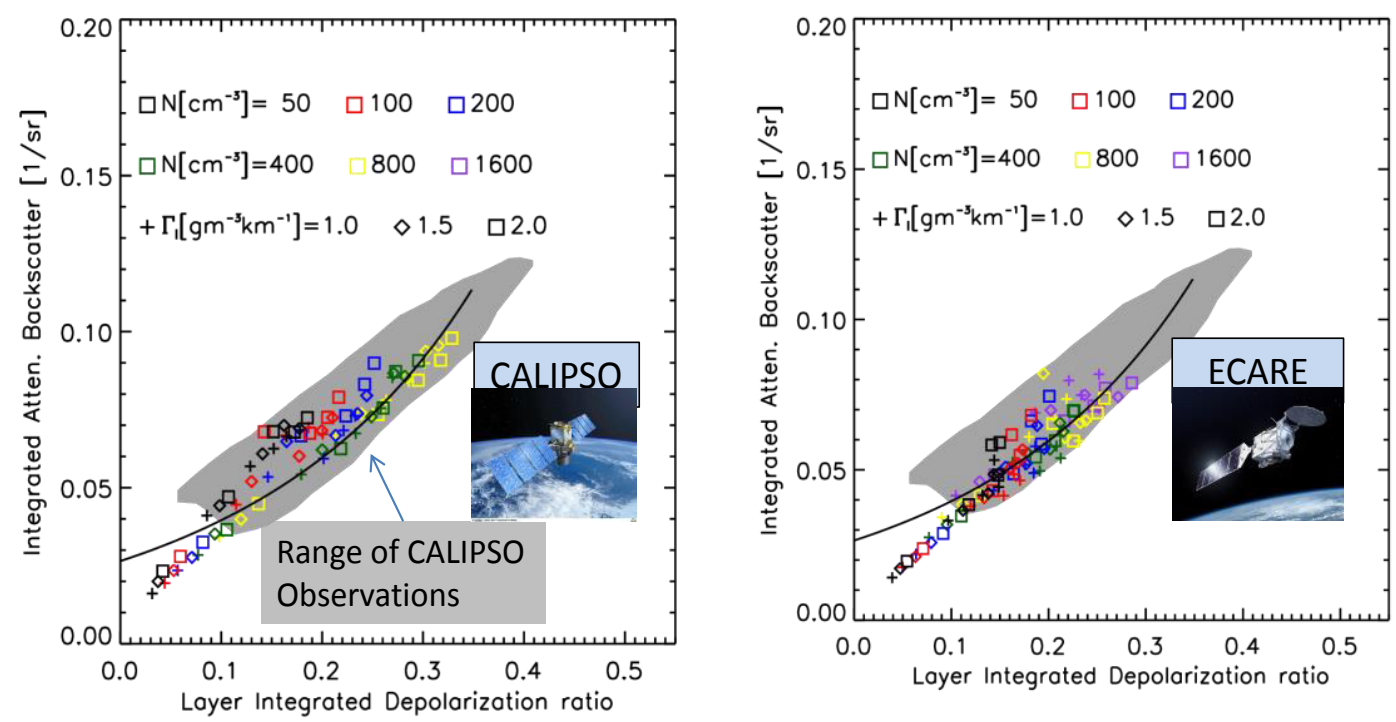

Figure 4: Integrated-layer-attenuated-backscatter vs. layer-depolarization for CALIPSO(Left) and ATLID (Right). Here the Grey-Area corresponds to the range of actual CALIPSO observations [7] and

the points correspond to MC results using the indicated liquid-water lapse-rates $\left(\Gamma_{l}\right)$ and cloud particle number concentrations (N). The solid lines correspond to Eq.(6) of [7].

cloud lidar return. This will be an important issue impacting, e.g. the retrieval of aerosol parameters below cirrus. On the other hand, the sensitivity to particle size may enable cirrus particle sizes to be estimated using the below cloud Rayleigh channel signal.

Calculations applied to idealized water clouds indicate that the layer-depolarization-vs-integrated attenuated backscatter relationship expected from ATLID will be similar to that observed by CALIPSO.

\section{ACKNOWLEDGEMENT}

Parts of this work were conducted as part of the ESA/ESTEC ATLAS project, contract No. 22638/09/NL/CT

\section{REFERENCES}

[1] Illingworth, I.J., et al., 2014: THE EARTHCARE SATELLITE: The next step forward in global measurements of clouds, aerosols, precipitation and radiation. Bull. Amer. Meteor. Soc., doi:10.1175/BAMS-D-12-00227.1.

[2] Platt, C. M. R., 1981: "Remote sounding of high clouds. III: Monte Carlo calculations of multiple-scattered lidar returns." J. Atmos.Sci., 38.1, 156-167.
[3] Hess M., R. Koelemeijer, P. Stammes 1998: Scattering matrices of imperfect hexagonal ice crystals, JQRT, doi:10.1016/S00224073(98)00007-7.

[4] Donovan, D.P. H. Klein Baltink, J. S. Henzing, S. R. de Roode, and A. P. Siebesma, 2015: A depolarisation lidar-based method for the determination of liquid-cloud microphysical properties, Atmos. Meas. Tech., 8, 237-266 doi:10.5194/amt-8-237-2015

[5] Voors, R., D.P. Donovan, J. Acarreta , M. Eisinger,; R. Franco, et al. 2007: "ECSIM: the simulator framework for EarthCARE", Proc. SPIE 6744, Sensors, Systems, and Next-Generation Satellites XI, 67441Y (October 17, 2007); doi: $10.1117 / 12.7377$

[6] Hogan, R., 2006: "Fast approximate calculation of multiply scattered lidar returns," Appl. Opt. 45, 5984-5992.

[7] Hu, Y., M. Vaughan, Z. Liu, B. Lin, P. Yang, D. Flittner, B. Hunt, R. Kuehn, J. Huang, D. Wu, S. Rodier, K. Powell, C. Trepte, and D. Winker, 2007: "The depolarization - attenuated backscatter relation: CALIPSO lidar measurements vs. theory," Opt. Express 15, 5327-5332. 